\title{
IDENTIFICAÇÃO DE POTENCIAIS INTERAÇÕES MEDICAMENTOSAS EM PRESCRIÇÕES DE PACIENTES DO CENTRO DE TERAPIA INTENSIVA DE UM HOSPITAL PRIVADO EM BELÉM-PA
}

José Eduardo Gomes Arruda ${ }^{1}$, Paulo Henrique de Aguiar Pinheiro², Chriscia Jamily Pinto de Sousa ${ }^{1}$, Maria Eleuziane dos Santos Silva ${ }^{2}$

${ }^{1}$ Universidade Federal do Pará, ${ }^{2}$ Centro Universitário Fibra

E-mail para correspondência: josearruda@ufpa.br Submetido em: 03/04/2020 e aprovado em: 23/06/2020

\begin{abstract}
RESUMO
Introdução: Os pacientes do Centro de Terapia Intensiva (CTI), geralmente, possuem comorbidades, politerapia e tempo de internação prolongado, motivos pelos quais necessitam da assistência de equipe multiprofissional. Nesse cenário, o farmacêutico intensivista se destaca como profissional capacitado para identificar problemas relacionados à terapia medicamentosa por meio da análise de prescrições e acompanhamento farmacoterapêutico. Objetivos: $\mathrm{O}$ presente estudo buscou identificar e classificar possíveis interações medicamentosas (IM) em pacientes hospitalizados no CTI de um hospital privado em Belém-PA, no período de janeiro a março de 2018. Métodos: Foram analisados prontuários clínicos de 45 pacientes hospitalizados. Para a identificação e classificação das IM, foi utilizada a base de dados Micromedex®. Resultados e discussão: Todos os pacientes possuíam mais de 60 anos, $51 \%$ eram do sexo masculino e $49 \%$ do sexo feminino. Foram analisadas 2.464 prescrições e identificadas 1.796 potenciais IM que foram classificadas como: 6,99\% contraindicadas, 60,37\% maior, 28,67\% moderada, 3,50\% menor e $0,47 \%$ desconhecida. A documentação referente às IM também foi classificada conforme a base de dados como $8,16 \%$ excelente, $69,23 \%$ regular, $22,14 \%$ boa e 0,47\% desconhecida. Dentre os 45 prontuários, apenas 1 não apresentou IM. Conclusões: Os resultados obtidos neste estudo auxiliam na avaliação das prescrições médicas de CTI, ressaltando a importância do farmacêutico intensivista a fim de contribuir com a equipe multiprofissional, visando reduzir os riscos provenientes da terapia medicamentosa e otimizar os resultados desejados.

Palavras-chaves: Interações Medicamentosas, Centro de Terapia Intensiva, Farmácia Clínica.
\end{abstract}




\begin{abstract}
ABSTRAT
Introduction: Patients at the Intensive Care Center (ICU) usually have comorbidities, polytherapies, and prolonged hospital stay, reasons why they need a multidisiplinary team assistance. In this scenario, pharmacist is a qualified professional to identify problems related to drug therapy by prescriptions and pharmacotherapeutic monitoring analysis. Aims: The present study aimed identify and classify possible drug interactions (DI) in patients hospitalized at an Intensive Care Center (ICU) of a private hospital in Belém-PA, from January to March 2018. Methods: 45 hospitalized patients were analysed using Micromedex ${ }^{\circledR}$ database for identification and classification of IM. Results and discussion: All patients were over 60 years old, $51 \%$ were male and $49 \%$ female. 2,464 prescriptions were analyzed and identified 1,796 potential DI, which were classified as: $6.99 \%$ contraindicated, $60.37 \%$ higher, $28.67 \%$ moderate, $3.50 \%$ lower and $0.47 \%$ unknown. DI documentation was also defined, according the database, as $8.16 \%$ excellent, $69.23 \%$ regular, $22.14 \%$ good, and $0.47 \%$ unknown. Among the 45 medical records, only one did not display DI. Conclusions: Results have assisted the evaluation of medical prescriptions from ICU, emphasizing the importance of pharmacists in intensive care in a multiprofessional team aiming to reduce risks of pharmacotherapy and optimizes expected results.
\end{abstract}

Keywords: Drug Interactions, Intensive Care Center, Clinical Pharmacy.

\title{
INTRODUÇÃO
}

O Centro de Terapia Intensiva (CTI) é a unidade hospitalar que oferece monitoramento e cuidado intensivo em tempo integral ao paciente e caracteriza-se pela alta tecnologia e complexidade da assistência realizada. Geralmente, os pacientes internados apresentam quadro clínico grave e consequente necessidade da politerapia, o que torna a farmacoterapia um fator de risco, podendo contribuir para o agravamento da sua evolução clínica ${ }^{(1)}$.

Devido às características e especificidades dos pacientes atendidos nessa unidade, o farmacêutico tem se especializado no atendimento ao paciente crítico, deixando de lado sua característica "generalista" e tornando-se farmacêutico intensivista ${ }^{(2)}$. Estudos mostram que os benefícios do farmacêutico inserido na equipe multidisciplinar do CTI têm reduzido os custos e diminuído a taxa de eventos adversos em até $78 \%{ }^{(3)}$.

A atuação do farmacêutico perpassa pelo acompanhamento farmacoterapêutico, identificação e manejo de eventos adversos a medicamentos, avaliação de incompatibilidades medicamentosas, além da análise da prescrição médica ${ }^{(1)}$. Segundo alguns estudos, as 
prescrições estão diretamente ligadas à maioria dos casos de erro de medicação, tornando-as a primeira barreira para a identificação de problemas relacionados à terapia, como, por exemplo, a ocorrência de interações medicamentosas (IM) ${ }^{(4)}$.

As IM constituem um evento clínico em que os efeitos de um fármaco são alterados pela presença de outro fármaco, alimento, bebida ou algum agente químico ambiental, representando a causa comum de efeitos adversos ${ }^{(5)}$. Dentre esses fatores de risco associados à sua ocorrência, destaca-se o número de medicamentos prescritos, já que foi evidenciado uma incidência de $80 \%$ de IM em pacientes que fazem politerapia. É válido ressaltar que a IM nem sempre resultam em dano ao paciente, porém a sua identificação, assim como o manejo das possíveis reações decorrentes delas, devem ser realizadas a fim de garantir a segurança do paciente, já que a grande maioria dessas IM são preveníveis ${ }^{(6)}$.

Nesse sentido, ao ser levado em conta a criticidade dos pacientes atendidos no CTI, assim como as particularidades de sua terapia, faz-se necessário caracterizar as IM com finalidade de diminuir os riscos relacionados ao uso de medicamentos, promovendo a segurança do paciente.

O presente artigo apresenta como objetivo principal detectar possíveis interações medicamentosas presentes nas prescrições médicas do CTI em um hospital privado de Belém.

\section{MÉTODOS}

O estudo foi observacional, transversal e retrospectivo, sendo desenvolvido no Hospital Adventista de Belém (HAB), uma entidade filantrópica privada em Belém-PA. Foram analisados prontuários de pacientes internados no CTI no período de janeiro a março de 2018. Para a identificação e caracterização de potenciais IM, foi utilizanda a base de dados Micromedex®. A análise estatística foi elaborada utilizando o software BioEstat $5.0^{(7)}$, sendo a significância estatística aceita de 95\% (p<0,05). Com relação aos aspectos éticos, este estudo seguiu a Resolução do Conselho Nacional de Saúde no 466, de 12 de dezembro de 2012, que regulamenta a pesquisa em seres humanos, sendo submetido à Plataforma Brasil sob CAAE $\mathrm{n}^{\circ}$ CAAE 91199218.0.000.8187 e aprovado via Parecer Consubstanciado $\mathrm{n}^{\circ}$ 2.756.817. Por se tratar de um estudo observacional, descritivo e retrospectivo, foi solicitada a dispensa do Termo de Consentimento Livre Esclarecido.

\section{RESULTADOS E DISCUSSÃO}

Foram incluídos no estudo, 45 pacientes. Todos eram idosos com média de idade de 82 ( \pm 7 ) anos, com prevalência do sexo feminino 51,1\% (11). O tempo de internação variou entre 
2 e 101 dias, com média de 21 dias (DP: \pm 23 ) de permanência. Durante o período de internação os pacientes receberam em média 55 prescrições, totalizando 2.464 prescrições médicas, 10.490 doses de medicamentos dispensados e 134 fármacos de princípios ativos diferentes. Em média, cada paciente recebeu 29 fármacos, sendo o mínimo 6 e o máximo 75. No período estudado, 12 (48\%) pacientes apresentaram como principal motivo de internação os CID: A41 (Outras Septicemias) e J18 (Pneumonia por microorganismo não especificado).

Entre os fármacos mais prescritos, encontram-se aqueles associados ao protocolo padrão da CTI: Pantoprazol (95,6\%), Dipirona (82,2\%), Enoxaparina (77,8\%), Midazolam (75,5\%), Insulina $(71,1 \%)$.

Os antimicrobianos foram prescritos para 39 pacientes (86,6\%). A utilização variou de 1 a 13 antimicrobianos diferentes por paciente, com média de 5,41. Dentre os mais prescritos estão: Piperacilina + Tazobactan (66,7\%), Ciprofloxacino (62,2\%), Meropenem (53,3\%), Amicacina (42,2\%), Polimixina B (37,8\%).

Em relação às IM, foram identificadas 1.796 potenciais em 44 (97,7\%) dos pacientes avaliados. O número de potencial de interações por paciente variou de 1 a 121, com média de 40,8 por paciente. As dez interações mais frequentes estão representadas na Tabela 1, destacando fentanil e midazolam (36 pacientes apresentaram a IM pelo menos uma vez) e Dipirona x Enoxaparina (32 pacientes apresentaram a IM pelo menos uma vez).

Tabela 1: Potenciais IM mais frequentes, classificadas de acordo com a gravidade e documentação.

\begin{tabular}{ccccc}
\hline Potenciais IM & Gravidade & Documentação & n & \% \\
\hline Fentanil x Midazolam & Maior & Regular & 36 & $81,8 \%$ \\
Dipirona x Enoxaparina & Maior & Bom & 32 & $72,7 \%$ \\
Bromoprida x Midazolam & Maior & Regular & 27 & $61,4 \%$ \\
Dipirona x Furosemida & Maior & Bom & 27 & $61,4 \%$ \\
Bromoprida x Fentanil & Maior & Regular & 25 & $56,8 \%$ \\
Midazolam x Tramadol & Maior & Regular & 25 & $56,8 \%$ \\
Dipirona x Hidrocortisona & Maior & Regular & 23 & $52,3 \%$ \\
Fentanil x Tramadol & Maior & Regular & 23 & $52,3 \%$ \\
Furosemida x Hidrocortisona & Moderada & Regular & 23 & $52,3 \%$ \\
Furosemida x Insulina regular & Moderada & Regular & 22 & $50,0 \%$ \\
Ciprofloxacino x Fentanil & Maior & Excelente & 21 & $81,8 \%$ \\
\hline
\end{tabular}

As frequências das ocorrências de IM de acordo com a classificação de gravidade e documentação estão representadas na Tabela 2. Do universo de 429 IM diferentes, cerca de 30 $(6,99 \%)$ foram classificadas como contraindicadas, enquanto $259(60,37 \%)$ foram classificadas 
como maior. A documentação mais prevalente foi a regular, responsável por $297(69,23 \%)$ das ocorrências, seguida da documentação classificada como boa em 95 dos casos $(22,14 \%)$.

Tabela 2: Interações medicamentosas potenciais de acordo com a classificação de gravidade, documentação.

\begin{tabular}{ccc}
\hline & GRAVIDADE & \\
\hline Classificação & Frequência & $\%$ \\
\hline Maior & 259 & $28,37 \%$ \\
Moderada & 123 & $3,50 \%$ \\
Menor & 15 & $6,99 \%$ \\
Contraindicada & 30 & $0,47 \%$ \\
Desconhecida & 2 & $\%$ \\
\hline Classificação & DOCUMENTAÇÃO & $8,16 \%$ \\
\hline Excelente & Frequência & $69,23 \%$ \\
Regular & 35 & $22,14 \%$ \\
Bom & 297 & $0,47 \%$ \\
Desconhecida & 95 & 2 \\
\hline
\end{tabular}

O teste de correlação de Pearson demonstrou que houve correlação significativa $(\mathrm{p}<0.05)$ entre as variáveis: interação medicamentosa $\mathrm{x}$ tempo de internação, número de interações $\mathrm{x}$ quantidade de fármacos e o tempo de internação x quantidade de fármacos (Tabela 3). Ao correlacionar a variável idade, não houve valores significativos.

Tabela 3: Matriz de correlação de Pearson entre as variáveis idade, número de interações, tempo de internação e quantidade de medicamentos.

\begin{tabular}{|c|c|c|c|}
\hline Matriz de Correlações & Idade & $\mathbf{N}^{\circ}$ de interações & Tempo de internação \\
\hline Temno de internacão & 0.092 & 0.679 & \\
\hline rempo de miternaçao & $0.546 \mathrm{~ns}$ & $0.0000 * *$ & \\
\hline Quantidade de medicamentos & $\begin{array}{r}-0.046 \\
0762 \mathrm{~ns}\end{array}$ & $\begin{array}{r}0.754 \\
00000 * *\end{array}$ & $\begin{array}{r}0.713 \\
00000 * *\end{array}$ \\
\hline
\end{tabular}

No presente estudo, destacou-se a prevalência de paciente idoso com idade média de 82 anos. Outras pesquisas demonstraram associação entre idosos e a presença de $\operatorname{IM}^{(8,9,10)}$. De acordo com a literatura, a idade é um fator de risco para o aumento de IM, uma vez que os idosos estão mais propensos a alterações fisiológicas, condições crônicas de saúde que 
favorecem uma permanência mais longa no CTI, maior uso de medicamentos por dia e o uso de uma farmacoterapia mais complexa ${ }^{(8,11)}$.

Com base nesse perfil, a amostra demonstrou similaridade entre o CTI estudado e outras unidades nacionais e internacionais. O estudo de realizado no CTI do Hospital Universitário Getúlio Vargas em Manaus (AM) ${ }^{(4)}$, descreveu resultados semelhantes quanto ao perfil dos pacientes no que se refere ao sexo e a idade, sem diferença significativa na proporção em função do sexo.

Conforme os dados obtidos no presente estudo, o principal motivo de internação foi o CID A41, denominado de "outras septicemias", seguido por J18 (Pneumonia por microrganismo não especificado), o que corrobora com o fato de $86,6 \%$ dos pacientes terem utilizado pelo menos um antimicrobiano. Conforme outra pesquisa ${ }^{(12)}$, as doenças infecciosas são mais propícias em idosos, e representam o principal motivo de agravo clínico que leva a internação no CTI. Além disso, ressalta-se que o hospital em que este estudo foi realizado possui certificado de acreditação em distinção em sepse, o que pode aumentar a identificação desta infecção.

A média de fármacos utilizados por paciente neste estudo foi de 29 . O elevado número de medicamentos prescritos para pacientes internados em CTI indica um aumento de risco, onde o número de medicamentos é diretamente proporcional ao desenvolvimento de IM, eventos adversos, elevação de custos, e tempo de internação ${ }^{(6,13)}$, o que poderia justificar o tempo médio de internação de 21 dias encontrado nesta pesquisa.

Neste estudo o midazolam + fentanil foi a IM mais frequente $(81,8 \%)$. Esse resultado também pôde ser observado em outra pesquisa ${ }^{(8)}$, que demonstrou que das 311 interações medicamentosas $40 \%$ estavam relacionadas a fármacos que atuam no sistema nervoso central, sendo a interação entre midazolam e fentanil a mais frequente. Considerando o efeito da associação desses dois fármacos pode-se ressaltar que o uso simultâneo de analgésicos opioides (fentanil) e benzodiazepínicos (midazolam) pode resultar em depressão respiratória aditiva (IM com gravidade maior). Em contrapartida, essa associação é comumente utilizada com base no sinergismo farmacológico, para fornecer conforto e alívio da ansiedade em pacientes em ventilação mecânica ${ }^{(14)}$.

Analisando o nível de documentação, foi possível perceber que a categoria mais frequente foi a regular. Logo, vale ressaltar que as informações sobre as interações não são acessíveis aos profissionais, visto que tais interações continuam frequentes e aquém de serem superadas. 
Nesse contexto, as interações contraindicadas deveriam ser evitadas ao máximo, porém isso se mostrou pouco frequente nesta pesquisa. Pode estar relacionado, entre outros motivos, a ausência do farmacêutico clínico na equipe multiprofissional do CTI, a fim de garantir maior segurança no uso de terapias medicamentosas, por meio do rastreamento de IM, identificação e prevenção de eventos adversos e erros de medicação, assim como a promoção da educação continuada, promovendo a comunicação entre os demais profissionais da equipe multidisciplinar, buscando assegurar qualidade e segurança na assistência, assim como, conforto e segurança ao paciente ${ }^{(15)}$.

\section{CONCLUSÕES}

Este estudo evidenciou que as prescrições em CTI possuem uma elevada frequência de potenciais IM, constatação já confirmada em outros estudos, resultante da grande quantidade de fármacos prescritos. Por esse motivo, sugere-se que se dê continuidade e maior enfoque a pesquisas sobre o tema, visando ampliar o conhecimento do profissional de saúde sobre interações e seus possíveis eventos adversos, possibilitando a criação de estratégias e protocolos que contribuam na melhoria da qualidade de vida do paciente crítico. Este estudo também ressalta a importância do farmacêutico intensivista, e a necessidade dele no ambiente do CTI, possibilitando a redução de erros relacionados à farmacoterapia medicamentosa, contribuindo, portanto, para o sucesso do tratamento, segurança do paciente e a eficácia terapêutica do indivíduo hospitalizado.

\section{REFERÊNCIAS BIBLIOGRÁFICAS}

1. Fideles GMA, Alcântara-Neto JM, Peixoto Júnior AA, et al. Pharmacist recommendations in an intensive care unit: Three-year clinical activities. Revista Brasileira de Terapia Intensiva. 2015; 27(2):149-154.

2. Alves NMC. Farmacêutico Intensivista: um novo profissional na UTI. João Pessoa: Sociedade Brasileira de Terapia Intensiva, 2012. Dissertação para obtenção do título de Mestre em Terapia Intensiva.

3. Kucukarslan SN, Peter M, Mlynareck M, et al. Pharmacists on rounding teams reduce preventable adverse drug events in hospital general medicine units. Arch. Intern. Med. 2003; 163(17):2014-18.

4. Reis WCT, Scopel CT, Correr CJ, et al. Analysis of clinical pharmacist interventions in a tertiary teaching hospital in Brazil. Einstein. 2013; 41(11):190-196. 
5. Secoli SR. Interações medicamentosas: fundamentos para a prática clínica da enfermagem. Revista da Escola de Enfermagem da USP. 2001; 35(1):28-34.

6. Cedraz SJMCD, Santos Junior MC. Identificação e caracterização de interações medicamentosas em prescrições médicas da unidade de terapia intensiva de um hospital público da cidade de Feira de Santana, BA. Rev. Soc. Bras. Clín. Méd. 2014; 75(55):1-7.

7. Ayres M., Ayres Junior M., Ayres D.L. \& Santos A.S. Bioestat 5.0 - Aplicações estatísticas nas áreas das ciências biomédicas. 5ª ed. Belém: Mamiraua; 2007.

8. Lima REF, Cassiani SHB. Potencial Drug Interactions in Intensive Care Patients at a Teaching Hospital. Rev Lat Am Enfermagem. 2009; 17(2):222-7.

9. Blix HS, Viktil KK, Moger TA, et al. Identification of drug interactions in hospitals -computerized screening vs. bedside recording. J Clin Pharm Ther. 2008; 33(2):131-9.

10. BleichI GW, Bleich A, Chiamulera P, et al. Frequency of potential interactions between drugs in medical prescriptions in a city in southern Brazil. São Paulo Medical Journal. 2009; 127(4):206-210.

11. Hammes JA, Pfuetzenreiter F, Silveira F, et al. Prevalência de potenciais interações medicamentosas droga-droga em unidades de terapia intensiva. Rev. bras. ter. intensiva. 2008; 20(4):349-354.

12. Barros LLS, Maia CSF, Monteiro MC. Fatores de risco associados ao agravamento de sepse em pacientes em Unidade de Terapia Intensiva. Cad. Saúde Colet. 2016; 24(4):388-396.

13. Piedade DV, Silva LAF, Valasques Junior GL, et al. Interações medicamentosas envolvendo antimicrobianos. Medicina. 2015; 48(3):295-307.

14. Bohomol E, Ramos LH, D’Innocenzo M. Medication errors in an intensive care unit. J Adv Nurs. 2009; 65(6):1259-67.

15. Finatto RB, Caon S. Análise das "quase falhas" no processo de prescrição detectadas pelo farmacêutico clínico. Revista Brasileira de Farmácia. 2015; 96(1):1042-1054. 